\title{
Can Trace-Weighted Images Be Used to Estimate Diffusional Kurtosis Imaging-Derived Indices of Non-Gaussian Water Diffusion in Head and Neck Cancer?
}

W e read with great interest the appealing article by Tu et al, ${ }^{1}$ aimed at assessing the role of histogram-based descriptors of the non-Gaussian diffusional kurtosis imaging (DKI) model ${ }^{2}$ in treatment-response prediction of nasopharyngeal carcinoma. They found that histogram-based analysis of DKI-derived indices of "kurtosis" and "diffusivity" may potentially predict the neoadjuvant chemotherapy and short-term radiation therapy response in locoregionally advanced nasopharyngeal carcinoma, therefore possibly modifying the treatment regimen. Considering that DKI is a quantitative diffusion MR imaging technique, we think it would be of practical interest to discuss in greater detail the approach used to estimate kurtosis and diffusivity in this study and in various DKI applications in general.

Tu et al ${ }^{1}$ used only 3 orthogonal diffusion-weighting directions as is usual in extracranial DKI studies. While a rigorous application of DKI would require the use of at least 15 diffusionweighting directions and 2 non-null b-values, ${ }^{2}$ this simplification (based on the assumption of a relatively low diffusion anisotropy degree for tumor lesions) allows reducing the scan time, which is a pivotal issue in numerous extracranial applications of diffusion MR imaging. In this context, the estimation of kurtosis and diffusivity as the average of directional kurtosis and diffusivity obtained separately along the 3 orthogonal directions (namely $\mathrm{K}$ and D) hence represents the best possible approximation ${ }^{3}$ for rotational invariant mean kurtosis (ie, the average of directional kurtosis along all spatial directions) and mean diffusivity, respectively. ${ }^{2}$ However, it is likely that Tu et $\mathrm{al}^{1}$ estimated $\mathrm{K}$ and $\mathrm{D}$ by fitting the DKI model to trace-weighted images (TWIs) (ie, the geometric means of acquired diffusion-weighted images (DWIs), along the 3 orthogonal diffusion-weighting directions), albeit not explicitly reported.

This fact represents an important methodologic issue that should be adequately discussed. Indeed, while the approach of using TWIs has been widely adopted in many extracranial applications of DKI, it is mathematically as well as theoretically incorrect and yields no advantage in terms of acquisition time. ${ }^{3,4}$ Notably, a simulation study has shown that this approach can potentially affect the estimation of $\mathrm{K}^{3}$ In particular, even for true $\mathrm{K}$ values $\sim 1$ (as typically observed in human tissue) and a low dif-

http://dx.doi.org/10.3174/ajnr.A6167 fusion anisotropy degree $(<0.2)$, the percentage error in the $\mathrm{K}$ estimation caused by the use of TWIs instead of single DWIs along the 3 orthogonal directions can range up to more than $30 \%$, resulting in greater uncertainty introduced by DWIs noise for typical signal-to-noise ratio values. In addition, a recent in vivo study has demonstrated that the fit of the DKI model to TWIs can introduce bias and error in the estimation of $\mathrm{K}$ and $\mathrm{D}$ of head and neck cancer, which can be non-negligible for single lesions. ${ }^{4}$ The median (95\% confidence interval) errors in $\mathrm{K}$ and $\mathrm{D}$ were $5.1 \%$ $(0.8 \%, 32.6 \%)$ and $1.7 \%(-2.5 \%, 5.3 \%)$, respectively.

In conclusion, toward an optimization of methods in quantitative DKI of the head and neck and extracranial regions in general, the use of at least 15 diffusion-weighting directions and 2 non-null b-values would be conceptually recommendable. ${ }^{2}$ Nonetheless, the widespread and growing tendency to obtain $\mathrm{K}$ and D by fitting the DKI model to TWIs should possibly be avoided because it can affect the estimation of DKI-derived indices. ${ }^{3,4}$

\section{REFERENCES}

1. Tu N, Zhong Y, Wang X, et al. Treatment response prediction of nasopharyngeal carcinoma based on histogram analysis of diffusional kurtosis imaging. AJNR Am J Neuroradiol 2019;40:326-33 CrossRef Medline

2. Jensen JH, Helpern JA. MRI quantification of non-Gaussian water diffusion by kurtosis analysis. NMR Biomed 2010;23:698-710 CrossRef Medline

3. Giannelli M, Toschi N. On the use of trace-weighted images in body diffusional kurtosis imaging. Magn Reson Imaging 2016;34:502-07 CrossRef Medline

4. Marzi S, Minosse S, Vidiri A, et al. Diffusional kurtosis imaging in head and neck cancer: on the use of trace-weighted images to estimate indices of non-Gaussian water diffusion. Med Phys 2018;45: 5411-19 CrossRef Medline

(1) M. Giannelli Unit of Medical Physics Pisa University Hospital “Azienda Ospedaliero-Universitaria Pisana” Pisa, Italy (1). Marzi Department of Electrical, Electronic, and Information Engineering "Guglielmo Marconi" University of Bologna Cesena, Italy 
(D) M. Mascalchi

Department of Clinical and Experimental Biomedical Sciences "Mario Serio"

University of Florence

Florence, Italy

(D). Diciotti

Department of Electrical, Electronic, and Information Engineering

"Guglielmo Marconi"

University of Bologna

Cesena, Italy

(D) C. Tessa

Unit of Radiology

Versilia Hospital

Lido di Camaiore, Italy 